\title{
Equivalence Relation between Initial Values and Solutions for Evolution $p$-Laplacian Equation in Unbounded Space
}

\author{
Liangwei Wang ${ }^{1}$, Jingxue Yin ${ }^{2, *}$ and Langhao Zhou ${ }^{1}$ \\ ${ }^{1}$ College of Mathematics and Statistics, Chongqing Three Gorges University, \\ Chongqing, 404000, China. \\ 2 School of Mathematical Science, South China Normal University, Guangzhou, \\ 510631, China.
}

Received 12 February 2020; Accepted 1 March 2020

\begin{abstract}
In this paper, an equivalence relation between the $\omega$-limit set of initial values and the $\omega$-limit set of solutions is established for the Cauchy problem of evolution $p$-Laplacian equation in the unbounded space $Y_{\sigma}\left(\mathbb{R}^{N}\right)$. To overcome the difficulties caused by the nonlinearity of the equation and the unbounded solutions, we establish the propagation estimate and the growth estimate for the solutions. It will be demonstrated that the equivalence relation can be used to study the asymptotic behavior of solutions.
\end{abstract}

AMS subject classifications: $35 \mathrm{~K} 55,35 \mathrm{~B} 40$

Key words: Asymptotic behavior, evolution $p$-Laplacian equation, unbounded function, propagation estimate, growth estimate.

\section{Introduction}

In this paper, we consider the asymptotic behavior of solutions for the Cauchy problem of the evolution $p$-Laplacian equation

\footnotetext{
${ }^{*}$ Corresponding author. Email addresses: wanglw08@163. com (L. Wang), yjx@scnu .edu.cn (J. Yin),
} zhoulanghao8@163.com (L. Zhou) 


$$
\begin{array}{ll}
\frac{\partial u}{\partial t}-\operatorname{div}\left(|\nabla u|^{p-2} \nabla u\right)=0 & \text { in } \mathbb{R}^{N} \times(0, \infty), \\
u(x, 0)=u_{0}(x) & \text { in } \mathbb{R}^{N},
\end{array}
$$

where $p>2$ and the nonnegative initial value

$$
u_{0} \in Y_{\sigma}\left(\mathbb{R}^{N}\right) \equiv\left\{\varphi \in C\left(\mathbb{R}^{N}\right): \lim _{|x| \rightarrow \infty}\left(1+|x|^{2}\right)^{-\frac{\sigma}{2}} \varphi(x)=0\right\}
$$

with $0 \leq \sigma<\frac{p}{p-2}$.

Since the beginning of this century, there has been a great interest in the complicate asymptotic behavior of solutions for some evolution equations [1-8]. To do this, a successful method is to establish the relation between the initial values and the solutions for the evolution equations in some Banach spaces. In 2002, it was Vázquez and Zuazua [9] who first considered the relation between the $\omega$-limit set of initial values and the $\omega$-limit set of solutions to the problem (1.1)(1.2) in the bounded space $L^{\infty}\left(\mathbb{R}^{N}\right)$. They found that the set of accumulation points of the rescaled solutions $u\left(t^{\frac{1}{p}} x, t\right)$ to the problem (1.1)-(1.2) in $L_{\text {loc }}^{\infty}\left(\mathbb{R}^{N}\right)$ as $t \rightarrow \infty$ coincides with the set of $\{S(1)(\varphi)\}$, where $\varphi$ ranges over the set of the accumulation points as $\lambda \rightarrow \infty$ of the family $\left\{u_{0}(\lambda x) ; \lambda>0\right\}$ in the weakstar topology of $L^{\infty}\left(\mathbb{R}^{N}\right)$. By using this relation, they proved that the complicated asymptotic behavior can happen in the solutions. Later Cazenave, Dickstein and Weissler [10-13] investigated the relation between the rescaled solutions $t^{\frac{\mu}{2}} u\left(t^{\beta} x, t\right)(\mu, \beta>0)$ and the initial values for the heat equation in bounded space $C_{0}\left(\mathbb{R}^{N}\right)$. They also used these relation to investigate the complicated asymptotic behavior of solutions. They also study the complicated asymptotic behavior of solutions for the Navier-Stokes equations and the Schrödinger equation $[14,15]$. In our recent papers $[16,17]$, we revealed that there exists an equivalence relation between the $\omega$-limit set of initial values and the $\omega$-limit set of rescaled solution$\mathrm{s} t^{\frac{\mu}{2}} u\left(t^{\beta} x, t\right)(\mu, \beta>0)$ in bounded space $C_{0}\left(\mathbb{R}^{N}\right)$, and use this relation to study the complicate asymptotic behavior of solutions for the Cauchy problem of the porous medium equation and the Cauchy problem of the evolution $p$-Laplacian equation respectively. The studies of other asymptotic behavior of solutions for the evolution equations can be found in [18-23].

Note that the relations in the above works are only considered in some bounded spaces. It follows from the existence theory for the evolution $p$-Laplacian equation that the solutions of the problem (1.1)-(1.2) are global even if the initial data belong to some unbounded spaces [24-26]. Our interest here is to study 
the relation between initial values and solutions for the problem (1.1)-(1.2) in the unbounded space $Y_{\sigma}\left(\mathbb{R}^{N}\right)$. The difficulties in our studies are mainly caused by the unbounded solutions and the nonlinearity of Eq. (1.1). Fortunately, we can establish the propagation estimate and the growth estimate for these unbounded solutions to overcome these difficulties. By using the properties of solutions in the unbounded space, we obtain that if

$$
u_{0} \in Y_{\sigma}^{+}\left(\mathbb{R}^{N}\right) \equiv\left\{\varphi \in Y_{\sigma}\left(\mathbb{R}^{N}\right) ; \varphi \geq 0\right\} \quad \text { with } \quad 0 \leq \sigma<\frac{p}{p-2}
$$

then

$$
\omega^{\sigma}\left(u_{0}\right)=S(1) \Omega^{\sigma}\left(u_{0}\right)
$$

where

$$
\begin{aligned}
& \omega^{\sigma}\left(u_{0}\right) \equiv\left\{f \in Y_{\sigma}\left(\mathbb{R}^{N}\right) ; \exists t_{n} \rightarrow \infty \text { s.t. } t_{n}^{-\frac{\sigma}{p-\sigma(p-2)}} u\left(t_{n}^{\frac{1}{p-\sigma(p-2)}} x, t_{n}\right) \rightarrow f(x) \text { in } Y_{\sigma}\left(\mathbb{R}^{N}\right)\right\}, \\
& \Omega^{\sigma}\left(u_{0}\right) \equiv\left\{\varphi \in Y_{\sigma}\left(\mathbb{R}^{N}\right) ; \exists \lambda_{n} \rightarrow \infty \text { s.t. } \lambda_{n}^{-\frac{2 \sigma}{p-\sigma(p-2)}} u_{0}\left(\lambda_{n}^{\frac{2}{p-\sigma(p-2)}} x\right) \rightarrow \varphi(x) \text { in } Y_{\sigma}\left(\mathbb{R}^{N}\right)\right\} .
\end{aligned}
$$

The relation (1.3) can be used to prove complicated asymptotic behavior of solutions to the problem (1.1)-(1.2). Since there exists an initial value $u_{0} \in B_{M}^{\sigma,+} \equiv\{\phi \in$ $\left.Y_{\sigma}^{+}\left(\mathbb{R}^{N}\right) ;\|\phi\|_{Y_{\sigma}\left(\mathbb{R}^{N}\right)} \leq M\right\}$ such that $\Omega^{\sigma}\left(u_{0}\right)=B_{M}^{\sigma,+}$, solutions of the problem (1.1)(1.2) can exhibit complicate asymptotic behavior by the relation (1.3), according to Vázquez and Zuazua [9].

The rest of this paper is organized as follows. In the next section, we give some definitions and properties. Section 3 is devoted to some estimates for the solution$\mathrm{s}$ of the problem (1.1)-(1.2) with the initial values $u_{0} \in Y_{\sigma}\left(\mathbb{R}^{N}\right)$. The equivalence relation is studied in Section 4 . Using the equivalence relation to investigate the asymptotic behavior is given in the last section.

\section{Preliminaries}

In this section we first introduce some concepts and give some propositions about the solutions to the problem (1.1)-(1.2).

Definition 2.1. ([24,25]) For $r>0, f \in L_{\text {loc }}^{1}\left(\mathbb{R}^{N}\right)$, let

$$
\||\varphi|\|_{r}=\sup _{R \geq r} R^{-\frac{N(p-2)+p}{p-2}} \int_{\{|x| \leq R\}}|\varphi(x)| \mathrm{d} x \quad \text { and } \quad \ell(\varphi)=\lim _{r \rightarrow \infty}\|\mid \varphi\|_{r} .
$$


The space $X_{0}$ is defined to be

$$
X_{0} \equiv\{\varphi \in X ; \ell(\varphi)=0\}
$$

with the norm $\||\cdot|\|_{1}$. Hence it is a Banach space.

If the initial value $u_{0} \in X_{0}$, the existence and uniqueness of global weak solution of the problem (1.1)-(1.2) had been proved in [24-26], and this solution satisfies the following proposition.

Proposition 2.1. $([25,26])$ The Cauchy problem of the evolution p-Laplacian equation (1.1)-(1.2) generates a continuous bounded semigroup in $X_{0}$ given by

$$
S(t): u_{0} \rightarrow u(x, t)
$$

In other words, $u(x, t)=S(t) u_{0} \in C\left([0, \infty) ; X_{0}\right)$. Moreover, if $u_{0} \in L^{q}\left(\mathbb{R}^{N}\right)$ with $1 \leq q \leq \infty$, then $S(t)$ is a contraction bounded semigroup in $L^{q}\left(\mathbb{R}^{N}\right)$.

The unbounded spaces $L^{\infty}\left(\rho_{\sigma}\right)$ and $Y_{\sigma}\left(\mathbb{R}^{N}\right)$ is defined as follows.

Definition 2.2. Let $0 \leq \sigma<\infty, \rho_{\sigma}(x)=\left(1+|x|^{2}\right)^{-\frac{\sigma}{2}}$. The weighted space $L^{\infty}\left(\rho_{\sigma}\right)$ is defined to be

$$
L^{p}\left(\rho_{\sigma}\right) \equiv\left\{\varphi \in L_{\text {loc }}^{1}\left(\mathbb{R}^{N}\right): \varphi \rho_{\sigma} \in L^{\infty}\left(\mathbb{R}^{N}\right)\right\}
$$

with $\|\varphi\|_{L^{\infty}\left(\rho_{\sigma}\right)}=\left\|\varphi \rho_{\sigma}\right\|_{L^{\infty}\left(\mathbb{R}^{N}\right)}$. The space $Y_{\sigma}\left(\mathbb{R}^{N}\right)$ is defined by

$$
Y_{\sigma}\left(\mathbb{R}^{N}\right) \equiv\left\{\varphi(x) \in C\left(\mathbb{R}^{N}\right): \lim _{|x| \rightarrow \infty} \varphi(x) \rho_{\sigma}(x)=0\right\}
$$

with the norm $\|\varphi\|_{Y_{\sigma}\left(\mathbb{R}^{N}\right)}=\left\|\varphi \rho_{\sigma}\right\|_{L^{\infty}\left(\mathbb{R}^{N}\right)}$.

It is easy to prove that $Y_{\sigma}\left(\mathbb{R}^{N}\right)$ and $L^{\infty}\left(\rho_{\sigma}\right)$ are Banach spaces.

Definition 2.3. Suppose that $u_{0} \in Y_{\sigma}\left(\mathbb{R}^{N}\right)$ with $0 \leq \sigma<\frac{p}{p-2}$. For $\varphi(x) \in L_{\text {loc }}^{1}\left(\mathbb{R}^{N}\right)$ and $\lambda>0$, let

$$
D_{\lambda}^{\sigma} \varphi(x) \equiv \lambda^{-\frac{2 \sigma}{p-\sigma(p-2)}} \varphi\left(\lambda^{\frac{2}{p-\sigma(p-2)}} x\right) .
$$

Then we define the limit set $\Omega^{\sigma}\left(u_{0}\right)$ by

$$
\Omega^{\sigma}\left(u_{0}\right) \equiv\left\{\varphi \in Y_{\sigma}\left(\mathbb{R}^{N}\right) ; \exists \lambda_{n} \rightarrow \infty \text { s.t. } D_{\lambda_{n}}^{\sigma}\left[u_{0}\right] \stackrel{w *}{\longrightarrow} f \text { in } Y_{\sigma}\left(\mathbb{R}^{N}\right) \text { as } n \rightarrow \infty\right\} \text {. }
$$

The $\omega$-limit set $\omega^{\sigma}\left(u_{0}\right)$ is defined to be

$$
\omega^{\sigma}\left(u_{0}\right) \equiv\left\{f \in Y_{\sigma}\left(\mathbb{R}^{N}\right) ; \exists t_{n} \rightarrow \infty \text { s.t. } D_{\sqrt{t_{n}}}^{\sigma}\left[S\left(t_{n}\right) u_{0}\right] \stackrel{n \rightarrow \infty}{\longrightarrow} f \text { in } Y_{\sigma}\left(\mathbb{R}^{N}\right)\right\},
$$

where $S(t)$ is the semigroup given in (2.1). 
For $\lambda>0$, the commutative relation between the semigroup operator $S(t)$ and the dilation operator $D_{\lambda}^{\mu, \beta} \varphi(x) \equiv \lambda^{\mu} \varphi\left(\lambda^{2 \beta} x\right)$

$$
D_{\lambda}^{\mu, \beta}\left[S\left(\lambda^{2} t\right) u_{0}\right]=S\left(\lambda^{2-2 p \beta-\mu(p-2)} t\right)\left[D_{\lambda}^{\mu, \beta} u_{0}\right]
$$

had been proven in $[7,17]$.

Definition 2.4. Let $d(x) \equiv \sup \left\{R ; u_{0}(y)=0\right.$ a.e. in $\left.B_{R}(x)\right\}$. The positive set of $u(x, t)$ at time $t$ is defined to be

$$
\Omega(t) \equiv\left\{x \in \mathbb{R}^{N} ; u(x, t)>0\right\} .
$$

The $\rho$-neighborhood of $\Omega(t)$ is given by

$$
\Omega_{\rho}(t) \equiv\left\{x \in \mathbb{R}^{N} ; d(x, \Omega(t))<\rho\right\},
$$

where $d(x, \Omega(t)) \equiv \sup \left\{R ; B_{R}(x) \cap \Omega(t)=\varnothing\right\}$.

\section{Some estimates}

To study the relation between initial values and solutions for the problem (1.1)(1.2) in unbounded space $Y_{\sigma}\left(\mathbb{R}^{N}\right)$, we need to give some estimates about the solutions first. The following lemma had been proven in [24,25].

Lemma 3.1. ([24,25]) Let $u(x, t)$ be the nonnegative weak solutions of the problem (1.1)(1.2). For given $x_{0} \in \mathbb{R}^{N}$, if

$$
B\left(x_{0}\right)=\sup _{R>0} R^{-\frac{N(p-2)+p}{p-2}} \int_{B_{R}\left(x_{0}\right)} u_{0}(y) \mathrm{d} y<\infty,
$$

where $B_{R}\left(x_{0}\right)=\left\{y ;\left|x_{0}-y\right|<R\right\}$, then

$$
u\left(x_{0}, t\right)=0 \text { for all } 0<t \leq C B\left(x_{0}\right)^{-(p-2)} .
$$

The following theorem concerns the propagation estimate for the solutions of the problem (1.1)-(1.2) with $u_{0} \in L^{\infty}\left(\rho_{\sigma}\right)$.

Theorem 3.1 (Propagation Estimate). Suppose $u_{0} \in L^{\infty}\left(\rho_{\sigma}\right)$ with $0 \leq \sigma<\frac{p}{p-2}$, then for $0 \leq t_{1} \leq t_{2}<\infty$, one can get

$$
\Omega\left(t_{2}\right) \subset \Omega_{\rho\left(t_{2}-t_{1}\right)}\left(t_{1}\right),
$$

where

$$
\rho(t)=C \max \left(\left\|u_{0}\right\|_{L^{\infty}\left(\rho_{\sigma}\right)}^{\frac{p-2}{p}} t^{\frac{1}{p}},\left\|u_{0}\right\|_{L^{\infty}\left(\rho_{\sigma}\right)}^{\frac{p-2}{p-\sigma(p-2)}} t^{\overline{p-\sigma(p-2)}}\right) .
$$


Proof. We only need to consider the case $t_{1}=0$. Suppose that $x_{0} \in \mathbb{R}^{N}$ with $d\left(x_{0}\right)>$ 0 . If $R<d\left(x_{0}\right)$, then the following equality holds

$$
\int_{B_{R}\left(x_{0}\right)} u_{0}(y) \mathrm{d} y=0
$$

and if $R \geq d\left(x_{0}\right)$, we deduce from Definition (2.1) and $0 \leq \sigma<\frac{p}{p-2}$ that

$$
\begin{aligned}
& R^{-\frac{N(p-2)+p}{p-2}} \int_{B_{R}\left(x_{0}\right)} u_{0}(y) \mathrm{d} y \\
= & R^{-\frac{N(p-2)+p}{p-2}} \int_{B_{R}\left(x_{0}\right)} u_{0}(y) \rho_{\sigma}(x)\left(1+|x|^{2}\right)^{\frac{\sigma}{2}} \mathrm{~d} y \\
\leq & \left\|u_{0}\right\|_{L^{\infty}\left(\rho_{\sigma}\right)} R^{-\frac{N(p-2)+p}{p-2}}\left(1+R^{2}\right)^{\frac{\sigma}{2}} \int_{B_{R}\left(x_{0}\right)} \mathrm{d} y \\
\leq & 2^{\frac{\sigma}{2}}\left\|u_{0}\right\|_{L^{\infty}\left(\rho_{\sigma}\right)} \max \left(R^{-\frac{p}{p-2}}, R^{-\frac{p}{p-2}+\sigma}\right) \\
\leq & 2^{\frac{\sigma}{2}}\left\|u_{0}\right\|_{L^{\infty}\left(\rho_{\sigma}\right)} \max \left(d\left(x_{0}\right)^{-\frac{p}{p-2}}, d\left(x_{0}\right)^{-\frac{p}{p-2}+\sigma}\right) .
\end{aligned}
$$

Consequently,

$$
\begin{aligned}
B\left(x_{0}\right) & =\sup _{R \geq d\left(x_{0}\right)} R^{-\frac{N(p-2)+p}{p-2}} \int_{B_{R}\left(x_{0}\right)} u_{0}(y) \mathrm{d} y \\
& \leq C\left\|u_{0}\right\|_{L^{\infty}\left(\rho_{\sigma}\right)} \max \left(d\left(x_{0}\right)^{-\frac{p}{p-2}}, d\left(x_{0}\right)^{-\frac{p}{p-2}+\sigma}\right)
\end{aligned}
$$

holds by (3.1). Then it follows from Lemma 3.1 that

$$
u\left(x_{0}, t\right)=0 \quad \text { for all } \quad 0 \leq t \leq C\left\|u_{0}\right\|_{L^{\infty}\left(\rho_{\sigma}\right)}^{-(p-2)} \min \left(d\left(x_{0}\right)^{p}, d\left(x_{0}\right)^{p-\sigma(p-2)}\right),
$$

and therefore

$$
\Omega(t) \subset \Omega_{\rho(t)}(0),
$$

where

$$
\rho(t)=C \max \left(\left\|u_{0}\right\|_{L^{\infty}\left(\rho_{\sigma}\right)}^{\frac{p-2}{p}} t^{\frac{1}{p}},\left\|u_{0}\right\|_{L^{\infty}\left(\rho_{\sigma}\right)}^{\frac{p-2}{p-\sigma(p-2)}} t^{\overline{p-\sigma(p-2)}}\right),
$$

and the proof is complete.

In the following theorem, we estimate the growth estimate of solutions $u(x, t)$ to the problem (1.1)-(1.2) with the initial value $u_{0} \in L^{\infty}\left(\rho_{\sigma}\right)$. 
Theorem 3.2 (Growth Estimate). Let $0 \leq \sigma<\frac{p}{p-2}$. If $0 \leq u_{0} \in L^{\infty}\left(\rho_{\sigma}\right)$, then there exists a constant $C$ such that

$$
0 \leq S(t) u_{0}(x) \leq C\left((1+t)^{\frac{2}{p-\sigma(p-2)}}+|x|^{2}\right)^{\frac{\sigma}{2}} \text { for } t>0 .
$$

That is,

$$
\left\|S(t) u_{0}\right\|_{L^{\infty}\left(\rho_{\sigma}\right)} \leq C(1+t)^{\frac{\sigma}{p-\sigma(p-2)}} .
$$

Moreover, if $0 \leq u_{0} \in Y_{\sigma}\left(\mathbb{R}^{N}\right)$, then

$$
S(t) u_{0} \in Y_{\sigma}\left(\mathbb{R}^{N}\right) \text { for } t \geq 0
$$

Proof. Consider the following problem

$$
\begin{array}{ll}
\frac{\partial u}{\partial t}-\Delta u^{m}=0 & \text { in } \mathbb{R}^{N} \times(0, \infty), \\
u(x, 0)=v_{0}(x)=M|x|^{\sigma} & \text { in } \mathbb{R}^{N} .
\end{array}
$$

For $\lambda>0$, let

$$
\lambda_{1}=\lambda^{\frac{p-\sigma(p-2)}{2 p}}, \quad \mu=-\frac{2 \sigma}{p-\sigma(p-2)}, \quad \beta=\frac{1}{p-\sigma(p-2)},
$$

then

$$
2-\mu(p-2)-2 p \beta=0 .
$$

It follows from the commutative relation (2.2) that

$$
\begin{aligned}
& \lambda^{-\frac{\sigma}{p}}\left[S\left(\lambda^{1-\frac{\sigma(p-2)}{p}} t\right) v_{0}\right]\left(\lambda^{\frac{1}{p}} x\right)=\lambda_{1}^{\mu}\left[S\left(\lambda_{1}^{2} t\right) v_{0}\right]\left(\lambda_{1}^{2 \beta} x\right) \\
= & S(t)\left[\lambda_{1}^{\mu} v_{0}\left(\lambda_{1}^{2 \beta} \cdot\right)\right](x)=S(t) v_{0}(x) .
\end{aligned}
$$

Letting $t=1, s=\lambda^{\frac{p-\sigma(p-2)}{p}}$ and $g(x)=S(1) v_{0}(x)$ in (3.4), one can get

$$
S(s) v_{0}(x)=s^{\frac{\sigma}{p-\sigma(p-2)}} g\left(s^{-\frac{1}{p-\sigma(p-2)}} x\right) .
$$

Since $v_{0} \in C\left(\mathbb{R}^{N}\right)$, we obtain from the regularity theory of the solutions that for $t>0$,

$$
0 \leq S(t) v_{0} \in C\left([0, \infty) \times \mathbb{R}^{N}\right)
$$


see $[24,26]$. Therefore (3.5) implies that that for $|x|=1$, the following limit holds:

$$
s^{\frac{\sigma}{p-\sigma(p-2)}} g\left(s^{-\frac{1}{p-\sigma(p-2)}} x\right)=S(s) v_{0}(x) \rightarrow v_{0}(x)=M|x|^{\sigma}=M
$$

as $s \rightarrow 0$. Put

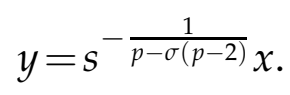

Observe that $|y| \rightarrow \infty$ as $s \rightarrow 0$. It follows from (3.6) that

$$
|y|^{-\sigma} g(y)-M \rightarrow 0 \quad \text { as } \quad|y| \rightarrow \infty
$$

Hence, there exists a nonnegative constant $C$ such that

$$
g(x) \leq C\left(1+|x|^{2}\right)^{\frac{\sigma}{2}}
$$

then we deduce from (3.5) that

$$
0 \leq S(s) v_{0}(x) \leq C\left(s^{\frac{2}{p-\sigma(p-2)}}+|x|^{2}\right)^{\frac{\sigma}{2}}
$$

therefore

$$
S(t) g(x)=S(t)\left[S(1) v_{0}\right](x)=S(t+1) v_{0}(x) \leq C\left((1+t)^{\frac{2}{p-\sigma(p-2)}}+|x|^{2}\right)^{\frac{\sigma}{2}} .
$$

Taking $\varphi(x)=M\left(1+|x|^{2}\right)^{\frac{\sigma}{2}}$, we thus obtain

$$
S(t) \varphi(x) \leq C\left((1+t)^{\frac{2}{p-\sigma(p-2)}}+|x|^{2}\right)^{\frac{\sigma}{2}} .
$$

Using Comparison Principle [24-26], we can get

$$
S(t) u_{0}(x) \leq C\left((1+t)^{\frac{2}{p-\sigma(p-2)}}+|x|^{2}\right)^{\frac{\sigma}{2}}
$$

if we take $M=\left\|u_{0}\right\|_{L^{\infty}\left(\rho_{\sigma}\right)}$ in (3.3).

We verify the second part of this theorem below. Note first that

$$
0 \leq u_{0} \in Y_{\sigma}\left(\mathbb{R}^{N}\right) \subset L^{\infty}\left(\rho_{\sigma}\right) .
$$

For given $t>0, R>0$, letting

$$
R(t)=R+1+C \max \left(\left\|u_{0}\right\|_{L^{\infty}\left(\rho_{\sigma}\right)}^{\frac{p-2}{p}} t^{\frac{1}{p}},\left\|u_{0}\right\|_{L^{\infty}\left(\rho_{\sigma}\right)}^{\frac{p-2}{p-\sigma(p-2)}} t^{\frac{1}{p-\sigma(p-2)}}\right)
$$


and then taking $\chi_{R+1}(x)$ be the cut-off function defined on $B_{R+1}$ relative to $B_{R}$, we deduce from Theorem 3.1 that

$$
\operatorname{supp}\left[S(t)\left(\chi_{R+1} u_{0}\right)\right] \subset\left\{x \in \mathbb{R}^{N} ;|x| \leq R(t)\right\} .
$$

This means that for $t, R>0$, the value of $S(t) u_{0}$ in $\mathbb{R}^{N} \backslash B_{R(t)}$ is only depended on the initial value $u_{0}$ in $\mathbb{R}^{N} \backslash B_{R+1}$, that is, if $|x|>R(t)$, then

$$
S(t)\left[\left(1-\chi_{R+1}\right) u_{0}\right](x)=S(t) u_{0}(x) .
$$

For every $\varepsilon>0$, it follows from the hypothesis $0 \leq u_{0} \in Y_{\sigma}\left(\mathbb{R}^{N}\right)$ that there exists a constant $R_{1}>1>0$ such that for $|x| \geq R_{1}$,

$$
\left(1+|x|^{2}\right)^{-\frac{\sigma}{2}} u_{0}(x)<\frac{\epsilon}{2}
$$

Letting $R=R_{1}$, we get

$$
\left(1-\chi_{R_{1}+1}\right) u_{0}(x)<\epsilon|x|^{\sigma}
$$

Putting $M=\epsilon$ in (3.3), one can get from (3.7) and Comparison Principle that there exists a constant $R_{2}$ such that if $|x|>R_{2}$, then

$$
S(t)\left[\left(1-\chi_{R_{1}+1}\right) u_{0}\right](x) \leq g(x) \leq 2 \epsilon|x|^{\sigma} .
$$

So (3.5) and (3.9) imply that if $|x| t^{-\frac{1}{p-\sigma(p-2)}}>R_{2}$, then

$$
S(t)\left[\left(1-\chi_{R_{1}+1}\right) u_{0}\right](x) \leq t^{\frac{\sigma}{p-\sigma(p-2)}} g\left(t^{-\frac{1}{p-\sigma(p-2)}} x\right) \leq 2 \epsilon|x|^{\sigma} .
$$

Combing (3.8) and (3.10), we get that if

$$
|x|>\max \left(R_{1}(t), t^{\frac{1}{p-\sigma(p-2)}} R_{2}\right),
$$

then

$$
S(t) u_{0}(x)=S(t)\left[\left(1-\chi_{R_{1}+1}\right) u_{0}\right](x) \leq 2 \epsilon|x|^{\sigma} .
$$

Observe that for $t>0$,

$$
S(t) u_{0}(x) \in L^{\infty}\left(\rho_{\sigma}\right) \text { and } S(t) u_{0}(x) \in C\left(\mathbb{R}^{N}\right),
$$

then it follows from (3.11) that for $t>0$,

$$
S(t) u_{0} \in Y_{\sigma}\left(\mathbb{R}^{N}\right),
$$

and the proof is complete. 


\section{Equivalence relation}

In this section, we study the relation between solutions and initial values of the problem (1.1)-(1.2) with initial value $u_{0} \in Y_{\sigma}^{+}\left(\mathbb{R}^{N}\right) \equiv\left\{\varphi \in Y_{\sigma}\left(\mathbb{R}^{N}\right) ; \varphi \geq 0\right\}$.

Theorem 4.1. Suppose $0 \leq \sigma<\frac{p}{p-2}$. If $u_{0} \in Y_{\sigma}^{+}\left(\mathbb{R}^{N}\right)$, then

$$
\omega^{\sigma}\left(u_{0}\right)=S(1) \Omega^{\sigma}\left(u_{0}\right) \equiv\left\{f: f=S(1) \varphi, \varphi \in \Omega^{\sigma}\left(u_{0}\right)\right\} .
$$

Proof. If $f \in \omega^{\sigma}\left(u_{0}\right)$, it follows from the definition of $\omega^{\sigma}\left(u_{0}\right)$ that there exists a sequence $t_{n} \stackrel{n \rightarrow \infty}{\longrightarrow} \infty$ such that

$$
\Gamma_{\sqrt{t_{n}}}^{\sigma}\left[S(1) u_{0}\right]=D_{\sqrt{t_{n}}}^{\sigma}\left[S\left(t_{n}\right) u_{0}\right]=S(1)\left[D_{\sqrt{t_{n}}}^{\sigma} u_{0}\right] \rightarrow f \quad \text { in } Y_{\sigma}\left(\mathbb{R}^{N}\right)
$$

Note that if $\lambda>1$ and $\phi \in Y_{\sigma}\left(\mathbb{R}^{N}\right)$, then

$$
\begin{aligned}
\left\|D_{\lambda}^{\sigma} \phi\right\|_{L^{\infty}\left(\rho_{\sigma}\right)} & =\sup _{x \in \mathbb{R}^{N}}\left(1+|x|^{2}\right)^{-\frac{\sigma}{2}} \lambda^{-\frac{2 \sigma}{\sigma(p-2)+p}} \phi\left(\lambda^{\frac{2}{\sigma(p-2)+p}} x\right) \\
& =\sup _{x \in \mathbb{R}^{N}}\left(1+\left|\lambda^{\frac{2}{\sigma(p-2)+p}} x\right|^{2}\right)^{-\frac{\sigma}{2}} \phi\left(\lambda^{\frac{2}{\sigma(p-2)+p}} x\right)\left(\frac{1+\left|\lambda^{\frac{2}{\sigma(p-2)+p}} x\right|^{2}}{\lambda^{\frac{2}{\sigma(p-2)+p}}\left(1+|x|^{2}\right)}\right)^{\frac{\sigma}{2}} \\
& \leq \sup _{x \in \mathbb{R}^{N}}\left(1+\left|\lambda^{\frac{2}{\sigma(p-2)+p}} x\right|^{2}\right)^{-\frac{\sigma}{2}} \phi\left(\lambda^{\frac{2}{\sigma(p-2)+p}} x\right)=\|\phi\|_{Y_{\sigma}\left(\mathbb{R}^{N}\right)}
\end{aligned}
$$

This means that

$$
\left\|D_{\sqrt{t_{n}}}^{\sigma} u_{0}\right\|_{Y_{\sigma}\left(\mathbb{R}^{N}\right)} \leq\left\|u_{0}\right\|_{Y_{\sigma}\left(\mathbb{R}^{N}\right)} \leq M
$$

for all $n \geq 1$. Hence there exists a subsequence $\left\{t_{n_{k}}\right\}$, which we still write as $\left\{t_{n}\right\}$, and a function $\varphi \in Y_{\sigma}\left(\mathbb{R}^{N}\right)$ such that

$$
D_{\sqrt{t_{n}}}^{\sigma} u_{0} \stackrel{w *}{\longrightarrow} \varphi \quad \text { in } Y_{\sigma}\left(\mathbb{R}^{N}\right)
$$

Consequently,

$$
\varphi \in \Omega^{\sigma}\left(u_{0}\right) \quad \text { and } \quad\|\varphi\|_{Y_{\sigma}\left(\mathbb{R}^{N}\right)} \leq\left\|u_{0}\right\|_{Y_{\sigma}\left(\mathbb{R}^{N}\right)} .
$$

For every $\epsilon>0$, applying Theorem 3.2 to $u_{0}$ and $\varphi$, we see that there exists a $R>0$ such that if $|x| \geq R$, then

$$
\left|S(1) u_{0}(x)\right|<\frac{\epsilon}{3} \quad \text { and } \quad|S(1) \varphi(x)|<\frac{\epsilon}{3} .
$$


Comparison Principle, (4.4) and (4.7) imply that if $x \geq R$, then

$$
\left|S(1)\left[D_{\sqrt{t_{n}}}^{\sigma} u_{0}\right](x)\right|<\frac{\epsilon}{3}
$$

for all $n \geq 1$. Taking

$$
R\left(u_{0}\right)=R+1+C \max \left(\left\|u_{0}\right\|_{Y_{\sigma}\left(\mathbb{R}^{N}\right)^{p}}^{\frac{p-2}{p}}\left\|u_{0}\right\|_{Y_{\sigma}\left(\mathbb{R}^{N}\right)}^{\frac{p-2}{p-\sigma(p-2)}}\right)
$$

and letting $\chi_{R\left(u_{0}\right)}(x)$ be the cut-off function defined on $B_{R\left(u_{0}\right)+1}$ relative to $B_{R\left(u_{0}\right)}$, we see that

$$
\begin{aligned}
& \operatorname{supp}\left[\left(1-\chi_{R\left(u_{0}\right)}\right) u_{0}\right] \\
\subset & \left\{x \in \mathbb{R}^{N} ;|x| \geq R+1+C \max \left(\left\|u_{0}\right\|_{Y_{\sigma}\left(\mathbb{R}^{N}\right)}^{\frac{p-2}{p}}\left\|u_{0}\right\|_{Y_{\sigma}\left(\mathbb{R}^{N}\right)}^{\frac{p-2}{p-\sigma(p-2)}}\right)\right\} .
\end{aligned}
$$

Then applying Theorem 3.1 to $\left(1-\chi_{R\left(u_{0}\right)}\right) u_{0}$, we get

$$
\operatorname{supp}\left[S(1)\left(1-\chi_{R\left(u_{0}\right)}\right) u_{0}\right] \subset\left\{x \in \mathbb{R}^{N} ;|x| \geq R+1\right\},
$$

hence

$$
\operatorname{supp} S(1)\left[\left(1-\chi_{R\left(u_{0}\right)}\right) u_{0}\right] \cap B_{R}=\varnothing .
$$

So apply Comparison Principle to get

$$
\operatorname{supp} S(1)\left[\left(1-\chi_{R\left(u_{0}\right)}\right) D_{\sqrt{t_{n}}}^{\sigma} u_{0}\right] \cap B_{R}=\varnothing .
$$

That is, for $x \in B_{R}$,

$$
S(1)\left(D_{\sqrt{t_{n}}}^{\sigma} u_{0}\right)(x)=S(1)\left[\chi_{R\left(u_{0}\right)}\left(D_{\sqrt{t_{n}}}^{\sigma} u_{0}\right)\right](x) .
$$

The same result holds for $S(1) \varphi$. That is, for $x \in B_{R}$,

$$
S(1) \varphi(x)=S(1)\left[\chi_{R\left(u_{0}\right)} \varphi\right](x) .
$$

Since $Y_{\sigma}\left(\mathbb{R}^{N}\right) \hookrightarrow \mathscr{S}^{\prime}\left(\mathbb{R}^{N}\right) \hookrightarrow \mathscr{D}^{\prime}\left(\mathbb{R}^{N}\right)$, (4.5) and (4.6) show that

$$
D_{\sqrt{t_{n}}}^{\sigma} u_{0} \rightarrow \varphi \quad \text { in } \mathscr{D}^{\prime}\left(\mathbb{R}^{N}\right)
$$

as $t_{n} \rightarrow \infty$. Observe that

$$
\chi_{R\left(u_{0}\right)} D_{\sqrt{t_{n}}}^{\sigma} u_{0}, \quad \chi_{R\left(u_{0}\right)} \varphi \in L^{1}\left(\mathbb{R}^{N}\right) .
$$


It now follows from (4.11) that

$$
\chi_{R\left(u_{0}\right)} D_{\sqrt{t_{n}}}^{\sigma} u_{0} \rightarrow \chi_{R\left(u_{0}\right)} \varphi \quad \text { in } L^{1}\left(\mathbb{R}^{N}\right)
$$

as $t_{n} \rightarrow \infty$. So for $0<\tau<1$, Proposition 2.1 implies that

$$
S(\tau)\left[\chi_{R\left(u_{0}\right)} D_{\sqrt{t_{n}}}^{\sigma} u_{0}\right] \rightarrow S(\tau)\left[\chi_{R\left(u_{0}\right)} \varphi\right] \quad \text { in } L^{1}\left(\mathbb{R}^{N}\right)
$$

as $t_{n} \rightarrow \infty$. This means that

$$
S(\tau)\left[\chi_{R(t)} D_{\sqrt{t_{n}}}^{\sigma} u_{0}\right] \rightarrow S(\tau)\left[\chi_{R(t)} \varphi\right] \quad \text { in } \mathscr{D}^{\prime}(\mathbb{R})
$$

as $t_{n} \rightarrow \infty$. By Theorem 3.2, (4.4) and (4.6) show that there exists a constant $C$ such that for all $n \geq 1$,

$$
\left\|S(\tau)\left[\chi_{R(t)} D_{\sqrt{t_{n}}}^{\sigma} u_{0}\right]\right\|_{Y_{\sigma}\left(\mathbb{R}^{N}\right)} \leq C,
$$

hence

$$
S(\tau)\left[\chi_{R(t)} D_{\sqrt{t_{n}}}^{\sigma} u_{0}\right] \stackrel{w *}{\longrightarrow} S(\tau)\left[\chi_{R(t)} \varphi\right] \quad \text { in } Y_{\sigma}\left(\mathbb{R}^{N}\right)
$$

as $t_{n} \rightarrow \infty$. By the regularity of the semigroup operators $S(t)$ and $0<\tau<1$, we obtain

$$
\left\|S(1)\left[\chi_{R(t)} D_{\sqrt{t_{n}}}^{\sigma} u_{0}\right]-S(1)\left[\chi_{R(t)} \varphi\right]\right\|_{Y_{\sigma}\left(\mathbb{R}^{N}\right)} \rightarrow 0
$$

as $t_{n} \rightarrow \infty$. So (4.9) and (4.10) imply that for all $x \in B_{R}$,

$$
\begin{aligned}
& \left(1+|x|^{2}\right)^{-\frac{\sigma}{2}}\left(S(1)\left[D_{\sqrt{t_{n}}}^{\sigma} u_{0}\right]-S(1)[\varphi]\right) \\
= & \left(1+|x|^{2}\right)^{-\frac{\sigma}{2}}\left(S(1)\left[\chi_{R(t)} D_{\sqrt{t_{n}}}^{\sigma} u_{0}\right]-S(1)\left[\chi_{R(t)} \varphi\right]\right) \rightarrow 0
\end{aligned}
$$

as $t_{n} \rightarrow \infty$. Then it follows from (4.7) and (4.8) that

$$
\left\|S(1)\left[D_{\sqrt{t_{n}}}^{\sigma} u_{0}\right]-f\right\|_{Y_{\sigma}\left(\mathbb{R}^{N}\right)}=\left\|S(1)\left[D_{\sqrt{t_{n}}}^{\sigma} u_{0}\right]-S(1)[\varphi]\right\|_{Y_{\sigma}\left(\mathbb{R}^{N}\right)} \rightarrow 0 .
$$

So we deduce from (4.2), (4.5) and (4.12) that

$$
f=S(1) \varphi \in S(1) \Omega^{\sigma}\left(u_{0}\right) .
$$

This means that

$$
\omega^{\sigma}\left(u_{0}\right) \subset S(1) \Omega^{\sigma}\left(u_{0}\right)
$$

On the other hand, suppose

$$
f \in S(1) \Omega^{\sigma}\left(u_{0}\right)
$$


Then by the definition of $S(1) \Omega^{\sigma}\left(u_{0}\right)$, there exists a function $\varphi \in \Omega^{\sigma}\left(u_{0}\right)$ such that

$$
f=S(1) \varphi \text {. }
$$

Hence there exists a sequence $\lambda_{n} \rightarrow \infty$ such that

$$
D_{\lambda_{n}}^{\sigma} u_{0} \rightarrow \varphi \text { in } Y_{\sigma}\left(\mathbb{R}^{N}\right)
$$

as $\lambda_{n} \rightarrow \infty$. Using a same proof of (4.12), we can get

$$
\left\|S(1)\left[D_{\lambda_{n}}^{\sigma} u_{0}\right]-f\right\|_{Y_{\sigma}\left(\mathbb{R}^{N}\right)}=\left\|S(1)\left[D_{\lambda_{n}}^{\sigma} u_{0}\right]-S(1)[\varphi]\right\|_{Y_{\sigma}\left(\mathbb{R}^{N}\right)} \rightarrow 0
$$

as $\lambda_{n} \rightarrow \infty$. Applying commutative relation (2.2) to $S(1)\left[D_{\lambda_{n}}^{\sigma} u_{0}\right]$, we have

$$
D_{\lambda_{n}}^{\sigma}\left[S(1) U_{0}\right]=S(1)\left[D_{\lambda_{n}}^{\sigma} u_{0}\right]
$$

Then taking $t_{n}=\lambda_{n}^{2}$ in (4.15), and using (4.16), we have

$$
D_{\lambda_{n}}^{\sigma}\left[S(1) u_{0}\right] \stackrel{t_{n} \rightarrow \infty}{\longrightarrow} f \quad \text { in } Y_{\sigma}\left(\mathbb{R}^{N}\right) .
$$

This means

$$
f \in \omega^{\sigma}\left(u_{0}\right)
$$

so (4.14) shows that

$$
S(1) \Omega^{\sigma}\left(u_{0}\right) \subset \omega^{\sigma}\left(u_{0}\right),
$$

therefore

$$
\omega^{\sigma}\left(u_{0}\right)=S(1) \Omega^{\sigma}\left(u_{0}\right)
$$

by (4.13), and the proof is complete.

\section{Complicated asymptotic behavior}

As an application of the relation (4.1), we follow the argument in [17] to prove that $\omega^{\sigma}\left(u_{0}\right)$ contain infinite functions. This result means that these solutions possess complicated asymptotic behavior, according to Vázquez and Zuazua [9].

Theorem 5.1. For $M>0$, let

$$
B_{M}^{\sigma,+} \equiv\left\{\varphi \in Y_{\sigma}\left(\mathbb{R}^{N}\right) ;\|\varphi\|_{Y_{\sigma}\left(\mathbb{R}^{N}\right)} \leq M \text { and } \varphi \geq 0\right\} .
$$

Then there exists $u_{0}(x) \in Y_{\sigma}\left(\mathbb{R}^{N}\right)$ such that

$$
\omega^{\sigma}\left(u_{0}\right)=S(1) B_{M}^{\sigma,+} \equiv\left\{f: f=S(1) \varphi, \varphi \in B_{M}^{\sigma,+}\right\} .
$$


Proof. Note that $B_{M}^{\sigma,+}$ with the weak-star topology is compact and separable. Let $\left\{\phi_{i}\right\}_{i=1}^{\infty}$ be a dense subset of $B_{M}^{\sigma,+}$. Given $\left\{\psi_{i}\right\} \subset B_{M}^{\sigma,+}$ such that for every $\phi_{i}$, there exists a subsequence $\left\{\psi_{i_{n}}\right\}$ of $\left\{\psi_{i}\right\}$ satisfying that

$$
\psi_{i_{n}}=\phi_{i}, \quad \forall i_{n} \geq 1
$$

Suppose

$$
\lambda_{i}= \begin{cases}a, & \text { if } i=1, \\ a^{i(p-\sigma(p-2))} \lambda_{i-1}, & \text { if } i>1,\end{cases}
$$

where $a>2$. Let $\chi_{i}(x)$ be the cut-off function defined on $E_{i} \equiv\left\{x \in \mathbb{R}^{N} ; a^{-i}<|x|<a^{i}\right\}$ relative to $E_{i-1} \equiv\left\{x \in \mathbb{R}^{N} ; a^{-i+1}<|x|<a^{i-1}\right\}$ and assume that

$$
u_{0}(x)=\sum_{i=1}^{\infty} D_{\lambda_{i}}^{-\sigma}\left(\chi_{i}(x) \psi_{i}(x)\right) .
$$

For $i>1$, it follows from (5.2) that

$$
\lambda_{i}^{\frac{2}{p-\sigma(p-2)}} a^{-i}=a^{i} \lambda_{i-1}^{\frac{2}{p-\sigma(p-2)}}>a^{i-1} \lambda_{i-1}^{\frac{2}{p-\sigma(p-2)}} .
$$

Since

$$
\operatorname{supp}\left(D_{\lambda_{i}}^{-\sigma}\left(\chi_{i}(x) \psi_{i}(x)\right) \subset\left\{x \in \mathbb{R}^{N} ; \lambda_{i}^{\frac{2}{p-\sigma(p-2)}} a^{-i}<|x|<\lambda_{i}^{\frac{2}{p-\sigma(p-2)}} a^{i}\right\},\right.
$$

we get that for $i \neq j$,

$$
\operatorname{supp} D_{\lambda_{i}}^{-\sigma}\left[\chi_{i}(x) \psi_{i}(x)\right] \cap \operatorname{supp} D_{\lambda_{j}}^{-\sigma}\left[\chi_{j}(x) \psi_{j}(x)\right]=\varnothing,
$$

hence the definition of $u_{0}$ implies that

$$
u_{0} \in B_{M}^{\sigma,+} .
$$

For every $\phi \in B_{M}^{\sigma,+}$, there exists a subsequence $\left\{\phi_{i_{n}}\right\}$ of $\left\{\phi_{i}\right\}$, which we also write as $\left\{\phi_{i}\right\}$, such that

$$
\phi_{i} \stackrel{w *}{\longrightarrow} \phi \quad \text { in } Y_{\sigma}\left(\mathbb{R}^{N}\right)
$$

as $i \rightarrow \infty$. For every $\phi_{i}, i \geq 1$, it follows from (5.3) that there exists a subsequence $\left\{\lambda_{i_{n}}\right\}$ of $\left\{\lambda_{i}\right\}$ such that

$$
D_{\lambda_{i_{n}}}^{\sigma} u_{0}=\phi_{i} \quad \text { in } A_{i_{n}-1}
$$


for all $i_{n} \geq 1$. Since $A_{i_{n}-1} \rightarrow \mathbb{R}^{N} \backslash\{0\}$ as $i_{n} \rightarrow \infty$, one can get that

$$
D_{\lambda_{i_{n}}}^{\sigma} u_{0} \stackrel{w *}{\longrightarrow} \phi_{i} \quad \text { in } Y_{\sigma}\left(\mathbb{R}^{N}\right)
$$

$i_{n} \rightarrow \infty$. By diagonal method, it follows from (5.5) that

$$
D_{\lambda_{i_{i}}}^{\sigma} u_{0} \stackrel{w *}{\longrightarrow} \phi \quad \text { in } Y_{\sigma}\left(\mathbb{R}^{N}\right)
$$

as $\lambda_{i_{i}} \rightarrow \infty$. This means that $\phi \in \Omega^{\sigma}\left(u_{0}\right)$, so

$$
B_{M}^{\sigma,+} \subset \Omega^{\sigma}\left(u_{0}\right)
$$

On the other hand, if $\phi \in \Omega^{\sigma}\left(u_{0}\right)$, it follows from the definition of $\Omega^{\sigma}\left(u_{0}\right)$ that there is a sequence $\lambda_{i} \rightarrow \infty$ such that

$$
D_{\lambda_{i}}^{\sigma} u_{0} \stackrel{w *}{\longrightarrow} \phi \quad \text { in } Y_{\sigma}\left(\mathbb{R}^{N}\right)
$$

Since $\left\|D_{\lambda_{i}}^{\sigma} u_{0}\right\|_{Y_{\sigma}\left(\mathbb{R}^{N}\right)} \leq\left\|u_{0}\right\|_{Y_{\sigma}\left(\mathbb{R}^{N}\right)} \leq M, \forall i \geq 1$ by (4.3), it follows from (5.7) that $\phi \geq 0$ and $\|\phi\|_{Y_{\sigma}\left(\mathbb{R}^{N}\right)} \leq M$. So $\phi \in B_{M}^{\sigma,+}$, this means that

$$
\Omega^{\sigma}\left(u_{0}\right) \subset B_{M}^{\sigma,+}
$$

hence

$$
\Omega^{\sigma}\left(u_{0}\right)=B_{M}^{\sigma,+}
$$

holds by (5.6). It follows from Theorem 4.1 and (5.8) that

$$
\omega^{\sigma}\left(u_{0}\right)=S(1) B_{M}^{\sigma,+}
$$

and the proof is complete.

\section{Acknowledgments}

This research was supported in part by NSFC (11771156 and 11371153), NSF of CQ (cstc2019jcyj-msxmX0381), Chongqing Municipal Key Laboratory of Institutions of Higher Education (Grant No. [2017]3), and Research project of Chongqing Three Gorges University (17ZP13). 


\section{References}

[1] T. Cazenave, F. Dickstein, F. B. Weissler, A solution of the constantcoefficient heat equation on $\mathbb{R}$ with exceptional asymptotic behavior: An explicit construction, Journal de Mathématiques Pures et Appliquées 85 (1) (2006) 119 - 150.

[2] T. Cazenave, F. Dickstein, F. B. Weissler, Nonparabolic asymptotic limits of solutions of the heat equation on $\mathbb{R}^{\mathrm{N}}$, Journal of Dynamics and Differential Equations 19 (3) (2007) 789-818.

[3] J. A. Carrillo, J. L. Vázquez, Asymptotic complexity in filtration equations, Journal of Evolution Equations 7 (2007) 471-495.

[4] J. L. Vázquez, M. Winkler, Highly time-oscillating solutions for very fast diffusion equations, Journal of Evolution Equations 11 (3) (2011) 725-742.

[5] L. W. Wang, J. X. Yin, Critical exponent for the asymptotic behavior of rescaled solutions to the porous medium equation, Electronic Journal of Differential Equations 10 (2017) 1-10.

[6] L. W. Wang, J. X. Yin, J. D. Cao, Remark on the Cauchy problem for the evolution p-Laplacian equation, Journal of Inequalities and Applications 175(1)(2017) 1-16.

[7] L. W. Wang, J. X. Yin, Classifications of asymptotic behavior of solutions for evolutionary $p$-Laplacian equation, Preprint, (2017) 1-38.

[8] L. W. Wang, J. X. Yin, Y. Zhou, Complicated asymptotic behavior of solutions for porous medium equation in unbounded space, Journal of Differential Equations 264 (10)(2018) 6302-6324.

[9] J. L. Vázquez, E. Zuazua, Complexity of large time behaviour of evolution equations with bounded data, Chinese Annals of Mathematics: Series B 23 (2) (2002) 293-310.

[10] T. Cazenave, F. Dickstein, F. B. Weissler, Universal solutions of a nonlinear heat equation on $\mathbb{R}^{\mathrm{N}}$, Annali Della Scuola Normale Superiore Di Pisa Classe Di Scienze 1 (1) (2003) 77-118.

[11] T. Cazenave, F. Dickstein, F. B. Weissler, Universal solutions of the heat equation on $\mathbb{R}^{N}$, Discrete and Continuous Dynamical Systems 9 (5)(2003) 1105-1132.

[12] T. Cazenave, F. Dickstein, F. B. Weissler, Multi-scale multi-profile global solutions of parabolic equations in $\mathbb{R}^{\mathrm{N}}$, Discrete and Continuous Dynamical Systems: Series $\mathrm{S}$ 5 (3) (2013) 449-472.

[13] H. Mouajria, S. Tayachi, F. B. Weissler, The heat semigroup on sectorial domains, highly singular initial values and applications, Journal of Evolution Equations 16 (2) (2016) 1-24.

[14] T. Cazenave, F. Dickstein, F. B. Weissler, Chaotic behavior of solutions of the NavierStokes system in $\mathbb{R}^{\mathrm{N}}$, Advances in Differential Equations 10 (4) (2006) 361-398.

[15] D. Y. Fang, J. Xie, T. Cazenave, Multiscale asymptotic behavior of the Schrödinger equation, Funkcialaj Ekvacioj 54 (54) (2011) 69-94.

[16] L. W. Wang, J. X. Yin, C. H. Jin, $\omega$-limit sets for porous medium equation with initial 
data in some weighted spaces, Discrete and Continuous Dynamical Systems-Series B 1 (1) (2013) 223-236.

[17] L. W. Wang, Relation between solutions and initial values for evolution $p$-Laplacian equation, Applied Mathematics Letters 69 (2017) 55-60.

[18] M. Bonforte, G. Grillo, Asymptotics of the porous media equation via Sobolev inequalities, Journal of Functional Analysis 225 (1) (2005) 33-62.

[19] J. L. Vázquez, Asymptotic behaviour for the porous medium equation posed in the whole space, Journal of Evolution Equations 3 (1) (2003) 67-118.

[20] F. Quirós, J. L. Vázquez, Asymptotic behaviour of the porous media equation in an exterior domain, Annali Della Scuola Normale Superiore Di Pisa Classe Di Scienze 9 (2) (1999) 183-227.

[21] J. A. Carrillo, J. L. Vázquez, Fine asymptotics for fast diffusion equations, Communications in Partial Differential Equations 28 (5-6) (2003) 1023-1056.

[22] C. Braendle, F. Quiros, J. L. Vázquez, Asymptotic behaviour of the porous media equation in domains with holes, Interfaces and Free Boundaries 9 (2)(2007)211-232.

[23] L. W. Wang, J. X. Yin, Proper spaces for the asymptotic convergence of solutions of porous medium equation, Nonlinear Analysis: Real World Applications 38 (2017) 261-270.

[24] DiBenedetto, E. and Herrero, M. A., On the Cauchy problem and initial traces for a degenerate parabolic equation, Transactions of the American Mathematical Society, 314(1) (1989)187-224.

[25] J. L. Vázquez, The porous medium equation: mathematical theory, Clarendon Press, Oxford University Press, 2007.

[26] J. L. Vázquez, Smoothing and decay estimates for nonlinear diffusion equations: Equations of Porous Medium Type, Oxford University Press, 2006. 